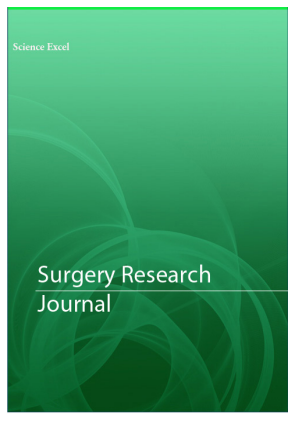

Correspondence

Ms Jennifer Mannion

Midland Regional Hospital, Tullamore Co Offaly Ireland, Tel: 00353872702904.

E-mail: JenniferMannion@rcsi.com

- Received Date: 05 June 2020;

- Accepted Date: 15 June 2020;

- Publication Date: 24 June 2020

Keywords

Inguinal Hernia, Classification

Copyright

(C) 2020 Science Excel. This is an openaccess article distributed under the terms of the Creative Commons Attribution 4.0 International license.

\section{Current classification of inguinal hernia: time to correct, update and change?}

\author{
Hehir Dermot J' and Mannion Jennifer ${ }^{2}$ \\ 'Adjunct Associate Professor, University of Limerick Graduate Entry Medical School, Ireland \\ ${ }^{2}$ Department of Surgery, Midland Regional Hospital, Tullamore Offaly, Ireland
}

\begin{abstract}
Repair of an inguinal hernia is a commonly performed surgical procedure. Current classifications are predominantly attributable to Hesselbach, who in the $19^{\text {th }}$ Century described the anatomy of the inguinal region and the relationship of inguinal hernia to the inferior epigastric vessels. However largely due to the dynamic visualization of inguinal anatomy at video-surgery, our better understanding of inguinal anatomy is facilitated; consequently, we believe that the inferior epigastric vessels have a coincidental anatomical relationship and have no significant causal relationship to groin herniation. Furthermore, we believe the current classification, using the terms 'direct' and 'indirect' are anatomically incorrect. It is our opinion that such classification should be abandoned in favour of a simplified system which reflects our enhanced appreciation of the anatomy.
\end{abstract}

\section{Introduction}

"Considering all that is written about the radical treatment of the inguinal hernia up until now, it can be somewhat risky to try to publish more about this subject " (Eduardo Bassini 1890 On the treatment of inguinal hernia)

It is estimated that $5 \%$ of the population will develop an inguinal hernia - the lifetime risk is $27 \%$ in males and $3 \%$ in females [1]. The cost to society is enormous, since inguinal hernia surgery is the most commonly performed intermediate surgical procedure. In the USA alone, $3 / 4$ million operations are performed annually [2]. Data from large multi - institution series suggest that tension free mesh repair is now the preferred modality [3]; debate continues whether mesh placement via open or minimally invasive (laparoscopic or robotic) techniques is optimal.

Proponents of each technique site advantages i.e. less pain [4], shorter hospital stay, early return to employment or disadvantages such as cost, learning curve, visceral injury [5].

\section{Anatomy}

Theinguinal canal, which in reality is the epicentre of inguinal hernia pathology, is an oblique area extending from the deep to superficial inguinal rings. The floor is formed by the inguinal ligament which extends between the anterior superior iliac spine laterally and the pubic tubercle medially it represents the reflected margin of the external oblique aponeurosis. The oblique nature of the canal is related to the insertion of the conjoint tendon, (comprising fibres from both the internal oblique and the transversus abdominus muscles), onto the pectineal line of the pubis [6].

The roof consists of an arch formed by the transversus abdominis muscle and lower fibres of internal oblique muscle. The anterior wall - as visualised at conventional open hernia repair is formed by the external oblique medially and the internal oblique laterally. The posterior wall is formed by the conjoint tendon (fibres of internal oblique / transversus abdominis).

The internal oblique muscle, with assistance from the transversus abdominis (collectively the conjoint tendon) is postulated to create a type of protective mechanism against inguinal hernia development $[7,8]$

The transversalis fascia is a potential bilayered wrapping created from infolding of the embryological umbilical funiculus [9].

\section{Aetiology of Inguinal Hernia}

More than two centuries ago (prior to the dynamic videoscopy era), Hesselbach recorded the inferior epigastric vessels as the definitive anatomical landmark with "direct" hernia medial to and "indirect" lateral to the vessels [10]. Thus, these terms historically relate to his belief that the herniated structure traverses the abdominal wall either directly or in an indirect oblique fashion via the inguinal canal .

The frequent coexistence of inguinal hernia in patients with collagen disorders [10], suggest a defect in the collagen fibre mechanism. Such pathology may explain the higher incidence of incisional hernia in aortic aneurysm surgery and defects associated with Ehlers - Danlos syndrome [11]. 
Historically, it was assumed that lateral "indirect" hernia were due to failure of obliteration of the embryological processus vaginalis in males; a similar process in females involves a delayed or incomplete closure of the canal of Nuck [12]. Approximately 10\% of children with observed asymptomatic patent processus vaginalis develop an inguinal hernia, in comparison to none of those in whom the process is obliterated [13]. The theory is further supported by adult data - patent processus vaginalis significantly increases the risk of post-operative inguinal hernia following robotic prostatectomy [14]. In contrast however, inguinal hernia may develop after closure of the processus vaginalis suggesting that the condition is in fact acquired [15]. Fruchaud hypothesised that groin hernia formation is due to the adoption of upright posture in modern man [13]. More recently, Radoievitch's angle between the ipsilateral inguinal ligament and a line traversing both anterior superior iliac spines has been associated with inguinal herniation due to abnormal pelvic anatomical angulation [16]. There is a documented increase in inguinal herniation with age, which may implicate muscle sarcopenia [17]. The occurrence of combined hernia (involving the medial inguinal fossa, internal inguinal ring and the supravesical fossa) is a common occurrence particularly in the elderly. These hernias appear to be progressive in nature and histopathological evaluation reveals fatty degeneration of myocytes with surrounding chronic inflammatory infiltrate often extending to involve all components of this area of the abdominal wall and associated structural damage to the inferior epigastric vessels. The author postulates that over time these pathological changes enables the progression to tricomponent herniation until the entire inguinal floor is devastated allowing a single protrusion to emerge [18].

\section{Discussion}

The internal oblique muscle forms at least a significant component of three of the four anatomical 'walls' of the inguinal canal. Thus a healthy internal oblique musculature is a major protective mechanism in the prevention of inguinal herniation. Damage to the internal oblique ie age related degeneration or collagen disorder, results in a global weakness in the inguinal region.

The proliferation of video facilitated groin hernia repair has facilitated our understanding of groin anatomy in vivo (Hesselbach did not have this luxury). It is now clearly possible to see internal defects in the inguinal abdominal wall and more importantly to demonstrate the global weakness associated with herniation.

The 'direct / indirect' classifications - such as Nyhus, Gilbert and Schumpelick are primarily based on anatomical localization of the hernia in conjunction with the size [19]. Other current classifications are based on findings attributable to Hesselbachs theory and therefore may not be appropriate for comparing data from different studies. Critics have argued they lack objectivity and prove difficult to remember leading to inconsistent usage [20].

It is our opinion that the terms 'direct' and 'indirect' for the description of inguinal hernias should be abandoned. We believe that a rather less specific description such as ' a two finger swelling in the medial aspect of the right groin' may be more appropriate.

\section{Conclusion}

The current classification of Inguinal herniation is based on historical anatomical dissection and for two centuries the inferior epigastric vessels have been unquestionably accepted as the distinction between 'direct' and 'indirect' inguinal herniation. It is our opinion that such classification should now be replaced, as it fails to appreciate our $21^{\text {st }}$ century knowledge of anatomy and the pathological processes involved in inguinal herniation.

\section{References}

1. Lichtenstein IL, Shulman AG, Amid PK, Montllor MM. The tension-free hernioplasty. Am J Surg. 1989;157(2):188-193.

2. Rutkow IM. Surgical operations in the United States. Then (1983) and now (1994). Arch Surg. 1997;132(9):983-990.

3. EU Hernia Trialists Collaboration. Mesh compared with nonmesh methods of open groin hernia repair: systematic review of randomized controlled trials. Br J Surg. 2000;87(7):854-859.

4. Kehlet $\mathrm{H}$, Bay-Nielsen $\mathrm{M}$; Danish Hernia Database Collaboration. Nationwide quality improvement of groin hernia repair from the Danish Hernia Database of 87,840 patients from 1998 to 2005 . Hernia. 2008;12(1):1-7.

5. McCormack K, Scott NW, Go PM, Ross S, Grant AM; EU Hernia Trialists Collaboration. Laparoscopic techniques versus open techniques for inguinal hernia repair. Cochrane Database Syst Rev. 2003;(1):CD001785.

6. Macready JFCH. A treatise on ruptures. 1893, Griffin \& Co.: London.

7. Skandalakis JE, Colborn GL, Androulakis JA, Skandalakis LJ, Pemberton LB. Embryologic and anatomic basis of inguinal herniorrhaphy. Surg Clin North Am. 1993;73(4):799-836.

8. Fortuny G, Rodríguez-Navarro J, Susín A, López-Cano M. Simulation and study of the behaviour of the transversalis fascia in protecting against the genesis of inguinal hernias. J Biomech. 2009;42(14):2263-2267.

9. Asakage N. Paradigm shift regarding the transversalis fascia, preperitoneal space, and Retzius' space. Hernia. 2018;22(3):499506.

10. Hesselbach FC. Neueste anatomisch-pathologische Untersuchungen über den Ursprung und da Fortschreiten der Leisten- und Schenkelbrüche 1814, Baumgartner: Würzburg

11. Read RC. Introduction. Hernia.2006;10(6):454-455.

12. Stavros AT, Rapp C. Dynamic ultrasound of hernias of the groin and anterior abdominal wall. Ultrasound Q. 2010;26(3):135-169.

13. Fruchaud H. Anatomie chirurgicale des hernies de l'aine . 1956, G Goin.: Paris.

14. Lee DH, Jung HB, Chung MS, Lee SH, Chung BH. Patent processus vaginalis in adults who underwent robot-assisted laparoscopic radical prostatectomy: predictive signs of postoperative inguinal hernia in the internal inguinal floor. Int J Urol. 2013;20(2):177-182.

15. Watanabe T, Yoshida F, Ohno M, et al. Morphology-based investigation of metachronous inguinal hernia after negative laparoscopic evaluation - is it acquired indirect inguinal hernia?. J Pediatr Surg. 2016;51(9):1548-1551.

16. Harissis HV, Georgiou GK. The role of pelvic bone anatomy in the pathogenesis of inguinal hernia. Chirurgia (Bucur). 2014;109(6):783-787.

17. Abramson JH, Gofin J, Hopp C, Makler A, Epstein LM. The epidemiology of inguinal hernia. A survey in western Jerusalem. J Epidemiol Community Health. 1978;32(1):59-67.

18. Amato G, Agrusa A, Rodolico V, et al. Combined inguinal hernia in the elderly. Portraying the progression of hernia disease. Int J Surg. 2016;33 Suppl 1:S20-S29.

19. Zollinger RM Jr. An updated traditional classification of inguinal hernias. Hernia. 2004;8(4):318-322.

20. Miserez M, Alexandre JH, Campanelli G, et al. The European hernia society groin hernia classification: simple and easy to remember [published correction appears in Hernia. 2008 Jun;12(3):335]. Hernia. 2007;11(2):113-116. 